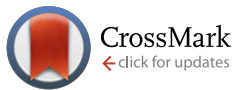

Cite this: Med. Chem. Commun., 2016, 7, 2192

\title{
Correction: Design and microwave assisted synthesis of novel 2-phenyl/2-phenylethynyl-3- aroyl thiophenes as potent antiproliferative agents
}

\author{
Rupinder Kaur Gill, ${ }^{\text {abc }}$ Ramandeep Kaur, ${ }^{a}$ Virender Kumar, ${ }^{d}$ Vivek Gupta, ${ }^{e}$ \\ Gagandeep Singh ${ }^{f}$ and Jitender Bariwal*ag
}

DOI: $10.1039 / \mathrm{c} 6 \mathrm{md} 90049 \mathrm{f}$

Correction for 'Design and microwave assisted synthesis of novel 2-phenyl/2-phenylethynyl-3-aroyl thiophenes as potent antiproliferative agents' by Rupinder Kaur Gill et al., Med. Chem. Commun., 2016, 7, 1966-1972.

www.rsc.org/medchemcomm

The authors regret the following errors in their paper: (1) The compound numbers shown below the structures in Table 1 should be corrected to show 10d and 12d, instead of 10a and 12a.

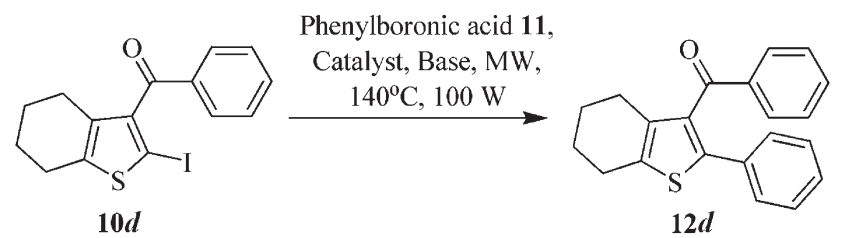

(2) On page 1967, section 2.1, first paragraph, when referring to optimization studies shown in Table 1 10a should be replaced by 10d and 12a should be replaced by 12d, as follows:

Earlier, we had reported the efficient formation of biaryl moiety via Suzuki-Miyaura cross-coupling reaction under microwave irradiation conditions; using tetrakis(triphenylphosphane)palladium $(0)\left[\operatorname{Pd}\left(\mathrm{PPh}_{3}\right)_{4}\right]$ as a catalyst and $\mathrm{Cs}_{2} \mathrm{CO}_{3}$ as a base under MWI at $140{ }^{\circ} \mathrm{C}$ and $100 \mathrm{~W}$ for $10 \mathrm{~min},{ }^{29}$ therefore, we have synthesized our targeted compound (4,5,6,7-tetrahydro-2phenylbenzo[ $b]$ thiophen-3-yl)(phenyl)methanone 12d by coupling of 2-iodo thiophene derivative 10d with phenylboronic acid 11 via Suzuki-Miyaura cross-coupling reaction (Scheme 1) by following the same protocol as employed earlier; however, the yield obtained was very low (Table 1, entry 1). Further, the optimization of this reaction under MW irradiation was carried out using $\mathrm{Pd}(\mathrm{OAc})_{2}$ and $\left[\mathrm{Pd}\left(\mathrm{PPh}_{3}\right)_{4}\right]$ as catalysts and $\mathrm{Cs}_{2} \mathrm{CO}_{3}$ and $\mathrm{K}_{2} \mathrm{CO}_{3}$ as bases in order to increase the yield of target compounds. It was observed that the use of $5 \mathrm{~mol} \%$ of $\mathrm{Pd}\left(\mathrm{PPh}_{3}\right)_{4}$ as a catalyst and 3.0 eq. of $\mathrm{K}_{2} \mathrm{CO}_{3}$ in $\mathrm{DMF}-\mathrm{H}_{2} \mathrm{O}$ under microwave irradiation (100 W) for $20 \mathrm{~min}$ at $140{ }^{\circ} \mathrm{C}$ furnished the desired compound $12 \mathrm{~d}$ in $91 \%$ yield (Table 1, entry 3).

The Royal Society of Chemistry apologises for these errors and any consequent inconvenience to authors and readers.

\footnotetext{
${ }^{a}$ Department of Pharmaceutical Chemistry, ISF College of Pharmacy, Moga-142001, Punjab, India

${ }^{b}$ I. K. Gujral Punjab Technical University, Kapurthala, Jalandhar-144 601, Punjab, India

${ }^{c}$ Department of Pharmaceutical Sciences, Guru Nanak Dev University, Amritsar-143 005, Punjab, India

${ }^{d}$ Department of Pharmaceutical Sciences, University of Nebraska Medical Center, Omaha, Nebraska, 68198 USA

${ }^{e}$ Post-Graduate Department of Physics \& Electronics, University of Jammu, Jammu Tawi-180 006, India

${ }^{f}$ Bio-Organic and Photochemistry Laboratory, Department of Pharmaceutical Sciences, Guru Nanak Dev University, Amritsar-143 005, Punjab, India

${ }^{g}$ Satiate Research \& Anatech Pvt. Ltd., HSIIDC, Barwala, Panchkula-134118, Haryana, India. E-mail: jitender.bariwal@gmail.com; Fax: +91 1636 239515;

Tel: +911636324200
} 